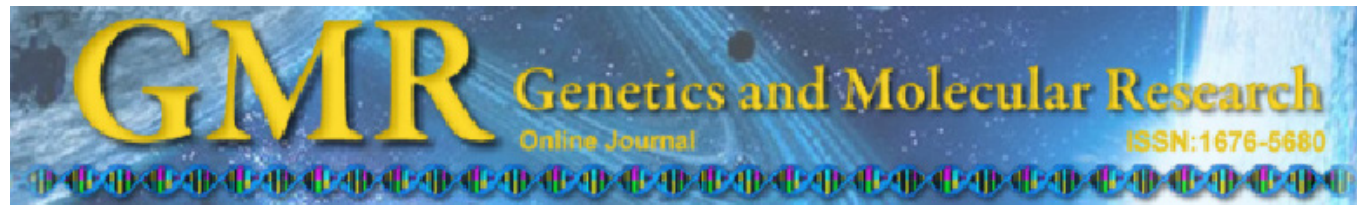

\title{
Recycling of superfine resolution agarose gel
}

\author{
T.-Y. Seng ${ }^{1,2}$, R. Singh ${ }^{3}$, Q.Z. Faridah ${ }^{2,4}$, S.-G. Tan ${ }^{4,5}$ and S.S.R.S. Alwee ${ }^{1}$ \\ ${ }^{1}$ Federal Land Development Authority Biotechnology Centre, FELDA \\ Agricultural Services Sdn. Bhd., Kuala Lumpur, Malaysia \\ ${ }^{2}$ Institute of Bioscience, Universiti Putra Malaysia, Serdang, Malaysia \\ ${ }^{3}$ Advanced Biotechnology and Breeding Centre, \\ Malaysian Palm Oil Board, Bandar Baru Bangi, Selangor, Malaysia \\ ${ }^{4}$ Biology Department, Faculty of Science, Universiti Putra Malaysia, \\ Serdang, Selangor, Malaysia \\ ${ }^{5}$ Department of Cell and Molecular Biology, \\ Faculty of Biotechnology and Biomolecular Sciences, \\ Universiti Putra Malaysia, Serdang, Selangor, Malaysia \\ Corresponding author: T.-Y. Seng \\ E-mail: ty.seng@felda.net.my
}

Genet. Mol. Res. 12 (3): 2360-2367 (2013)

Received February 2, 2012

Accepted September 25, 2012

Published March 11, 2013

DOI http://dx.doi.org/10.4238/2013.March.11.1

ABSTRACT. Genetic markers are now routinely used in a wide range
of applications, from forensic DNA analysis to marker-assisted plant
and animal breeding. The usual practice in such work is to extract the
DNA, prime the markers of interest, and sift them out by electrically
driving them through an appropriate matrix, usually a gel. The gels,
made from polyacrylamide or agarose, are of high cost, limiting
their greater applications in molecular marker work, especially in
developing countries where such technology has great potential. Trials
using superfine resolution (SFR) agarose for SSR marker screening
showed that it is capable of resolving SSR loci and can be reused up to
14 times, thus greatly reducing the cost of each gel run. Furthermore,
for certain applications, low concentrations of agarose sufficed and
switching to lithium borate buffer, instead of the conventional Tris-
borate-ethylenediaminetetraacetic acid buffer, will further save time
and cost. The $2.5 \%$ gel was prepared following the Agarose SFR 
manual by adding $2.5 \mathrm{~g}$ agarose powder into $100 \mathrm{~mL} 1 \mathrm{X}$ lithium borate buffer in a $250-\mathrm{mL}$ flask with rapid stirring. Two midigels $(105 \times 83$ $\mathrm{mm}, 17$ wells) or 4 minigels ( $50 \times 83 \mathrm{~mm}, 8$ wells), $4 \mathrm{~mm}$ thickness can be prepared from $100 \mathrm{~mL}$ gel solution. A total of 1680 PCR products amplified using 140 SSR markers from oil palm DNA samples were tested in this study using SFR recycled gel. As average, the gel can be recycled 8 times with good resolution, but can be recycled up to 14 times before the resolutions get blurred.

Key words: Superfine resolution; Agarose gel; Recycling; Microsatellite markers (SSR)

\section{INTRODUCTION}

Rapid advances of techniques in molecular genetics have been closely followed by increased use of their methods and result in routine applications. Mapping and the use of molecular markers - pieces of DNA that enable genetic differentiation and gene tagging were among the 1st benefits of such advances. DNA markers are now commonly used in DNA forensic science, for example, and are routinely present in the suite of tools used by plant and animal breeders, taxonomists, and conservationists.

Briefly, the use of DNA markers involves extracting DNA from a selected candidate or sample, priming its variously sized markers, and separating them on a matrix. The matrix is usually a gel, which allows migration of the markers under an applied voltage. Most early methods used polyacrylamide (PA) gel, but it is cumbersome to prepare, toxic, and costly; therefore, simpler, cheaper, and non-toxic agarose, from seaweed has been preferred for many applications (White and Kusukawa, 1997; Guilliatt, 2002; Gaudet et al., 2007; Eschholz et al., 2008; Raji et al., 2009). However, the lower separation capacity of the latter, usually for size differences of $<4 \mathrm{bp}$, is a drawback in some applications (Perumal et al., 2008). Proprietary preparations improved resolutions: for example, superfine resolution (SFR) gel (Agarose SFR ${ }^{\mathrm{TM}}$, Amresco, Solon, OH, USA; www.amresco-inc.com) is a highresolution sieving agarose that rivals PA in its capability for resolving DNA fragments of $<1000$ bp (White and Kusukawa, 1997). DNA bands differing in size by only $2 \%$ can be resolved in the range of 100-1000 bp (Boffey, 1984; Agarose SFR ${ }^{\mathrm{TM}}$ product information, Amresco). Compared with PA, SFR gel is easy to use and can be rapidly prepared, although it is technically more demanding to do so.

Cheaper agarose, including SFR preparations, will likely be in greater demand in developing countries in which the cost of gel limits more widespread use of molecular markers (Routman and Cheverud, 1994; Palacios et al., 2000). Large quantities of gel are required to analyze markers for genome mapping, primer screening, examination of total DNA, and other applications (Wang et al., 2003). However, gel cost can be reduced if the agarose can be reused - up to 14 times, as reported in this paper. In this investigation on recycling gels, other cost- and time-saving measures, such as using lower concentrations of agarose in gels for certain applications, using lithium borate (LB) buffer instead of the conventional Trisacetate-ethylenediaminetetraacetic acid (TAE)/Tris-borate-ethylenediaminetetraacetic acid (TBE) buffers, and orange-G instead of blue dyes, were also explored. 


\section{MATERIAL AND METHODS}

We used Agarose $\mathrm{SFR}^{\mathrm{TM}}$ (Amresco), a high-resolution sieving agarose with low melting temperature. Manufacturer specifications were as follows: ash $\leq 0.35 \%$; conductivity $(1 \%$, in water) at $25^{\circ} \mathrm{C} \leq 10 \mu \mathrm{mhos}$; electroendosmosis $\leq 0.12$ (-Mr); gel strength $(1.5 \%$, water) $>500 \mathrm{~g} / \mathrm{cm}^{2}$; gelling temperature $(1.5 \%$, water $) \leq 30.0^{\circ} \mathrm{C}$; melting point $(1.5 \%$, water $) \leq 70.0^{\circ} \mathrm{C}$; moisture $\leq 7.0 \% ; \mathrm{pH} \approx 7.0$ ( $1 \%$, water) at $25^{\circ} \mathrm{C}$; and sulfate $\leq 0.11 \%$.

For convenience, the buffer was a proprietary LB preparation (Faster Better Media LLC, Hunt Valley, MD, USA); however, it can be prepared in the laboratory from lithium hydroxide monohydrate and boric acid. LB buffer was tested, instead of TAE/TBE as recommended in the Agarose SFR ${ }^{\mathrm{TM}}$ manual, because it is cheaper and heats up less at higher temperatures, allowing higher voltages to be used for faster gel runs. To prepare the gel, 2.5 $\mathrm{g}$ agarose powder was added to $100 \mathrm{~mL} 1 \mathrm{X} \mathrm{LB}$ buffer in a $250-\mathrm{mL}$ flask with rapid stirring. The flask was marked to the level of the agarose. Once thoroughly wet, the agarose was microwaved at high power. Melting agarose can trap considerable air and boil over quickly; therefore, the mixture was watched carefully and removed from the microwave oven at incipient boiling, at which point gentle stirring was continued manually to aid the mixing without trapping more air. The procedure was repeated until the powder melted fully and no more bubbles appeared in the agarose solution. The volume of buffer lost through evaporation was replaced by adding $1 \mathrm{X}$ LB buffer to the level marked on the flask.

The agarose solution was then cooled under a tap while being continuously stirred manually. When the bottom of the flask cooled sufficiently to be held, $33 \mathrm{~mL}$ was poured into a casting tray $(8.2 \times 11 \mathrm{~cm},<4 \mathrm{~mm}$ thick $)$ and left to solidify at room temperature for 20 $\mathrm{min}$. The gel was then submerged in electrophoresis buffer in a horizontal electrophoresis apparatus. The polymerase chain reaction (PCR) products were mixed with orange-G loading dye and loaded into the wells. Although bromophenol blue and xylene cyanine blue are commonly used dyes, we tested orange-G because it is easier to elute when the gel is recycled. Gel electrophoresis was carried out for $40 \mathrm{~min}$ at a constant $180 \mathrm{~V}$ using $1 \mathrm{X}$ LB buffer. The gel was then stained with $0.5 \mu \mathrm{g} / \mathrm{mL}$ ethidium bromide (EtBr) for $2-5 \mathrm{~min}$, de-stained in distilled water for 15-20 min, and viewed under an ultraviolet (UV) transilluminator light. Images were captured with a UVIpro Silver Gel Documentation System (UVITEC, Cambridge, UK), processed, and stored using UVIsoft Image Acquisition and the Analysis software version 12.1 for Windows ${ }^{\circledR}$ (UVITEC).

\section{Recycling of $2.5 \%(w / v)$ SFR gel}

After the gel was photographed, it was crumpled and placed in the flask previously used to prepare it, and 1X LB buffer was added. The crumpled gel was soaked in the buffer for $24 \mathrm{~h}$ to elute most of the PCR products, the orange-G dye, and some of the EtBr. The buffer was then decanted and the gel re-melted in the microwave oven. The level of the agarose solution was maintained at the mark on the flask by adding 1 X LB buffer to replenish that lost through evaporation. The rest of the procedure was the same as that reported above for gel preparation. Re-melting and re-pouring were carried out under a fume hood owing to the EtBr retained in the gels. 


\section{RESULTS}

Figure 1 shows photographs of gel runs of the same amplified PCR products using 2 media: PA and SFR gels. The comparison confirms that SFR gel sufficed for most primer screening purposes, with nearly the same resolution as that of PA, which required silver staining.

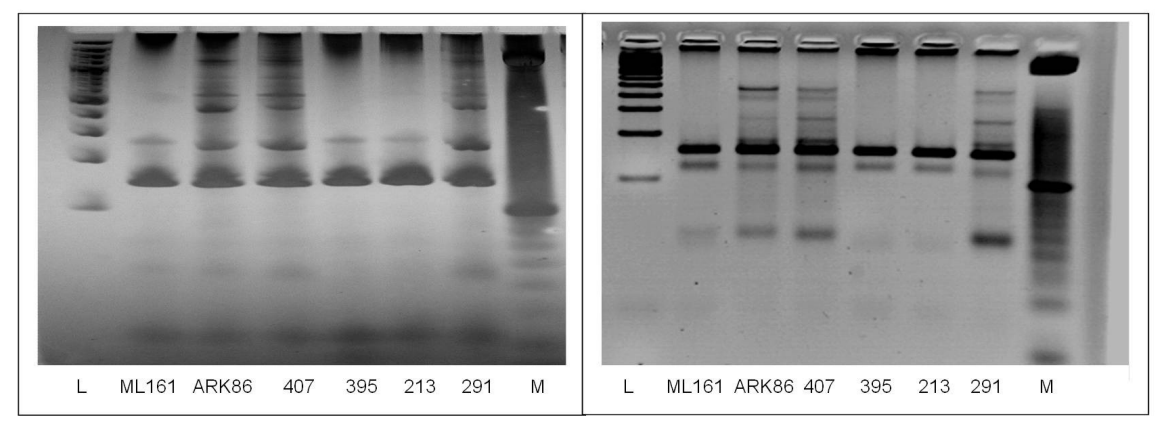

A

B

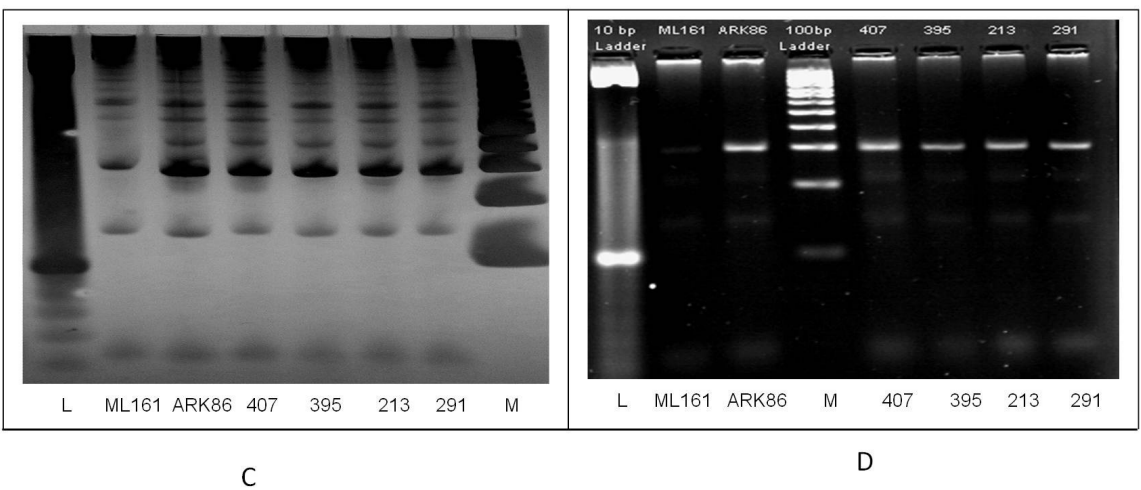

Figure 1. Separation of amplification products using SSR primers on $6 \%$ polyacrylamide (PA) and 3\% superfine resolution (SFR) agarose gels. A. C. Amplification products from single-locus DNA microsatellite primer pairs CIR 377 and 391 of the same 6 individuals were separated on 6\% PA and 3\% SFR agarose gels B. D. PCRs, electrophoretic conditions and post-electrophoretic stainings were as described in the text. All gels used 100-bp (lane L) and 10-bp (lane M) Ladder (New England Biolabs) as size standards.

Figures 2 and 3 show bands that appeared after repeated runs of a fresh gel $(2.5 \%$ (w/v) SFR agarose). Clear resolution of the amplified PCR products (100-350 bp) and DNA ladders can be seen. Simple sequence repeat allelic bands - the most intensely stained bands - were distinctly resolved with faintly stained stutter (artifactual) bands above and below the allelic bands in the amplified products.

The background fluorescence increased progressively with the number of runs, as the resolution of the fragments decreased owing to dust and sediment accumulation. To overcome this accumulation, the gel apparatus and documentation viewer were wiped clean before each run or visualization, and the melted recycled gel was filtered using Kimtech Science KimWipes delicate task wipers (Kimberly-Clark Corporation, Irving, TX, USA). 


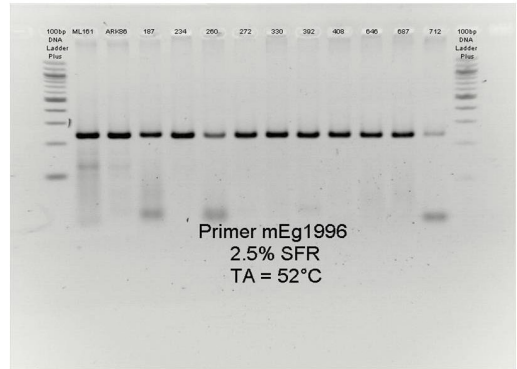

A

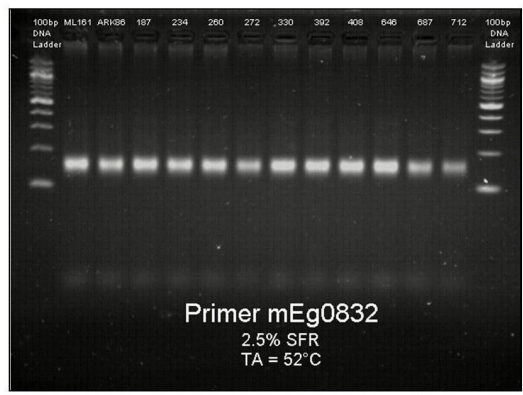

C

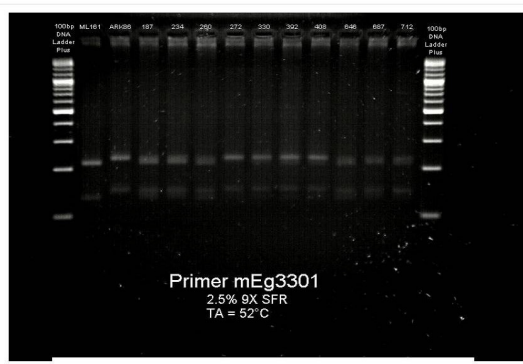

$\mathrm{E}$

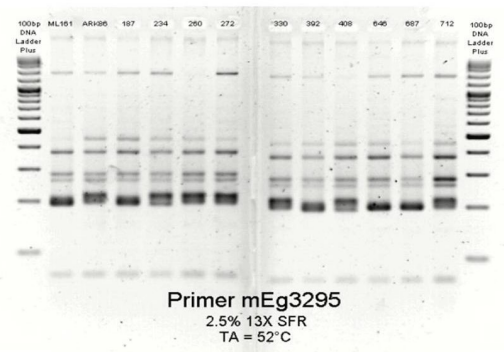

G

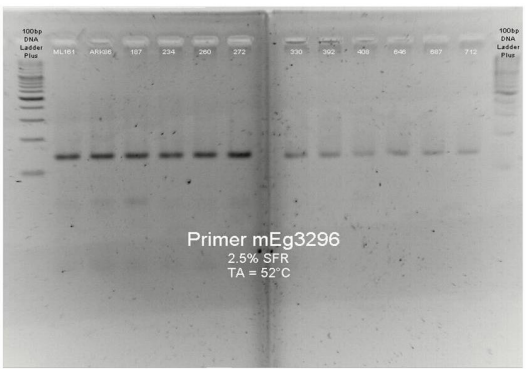

B

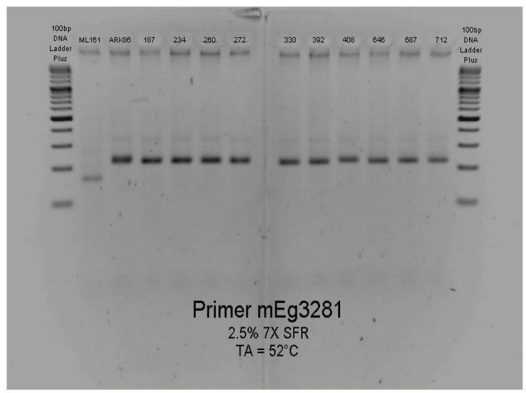

D

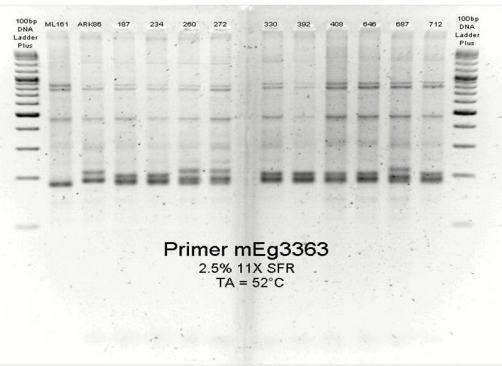

F

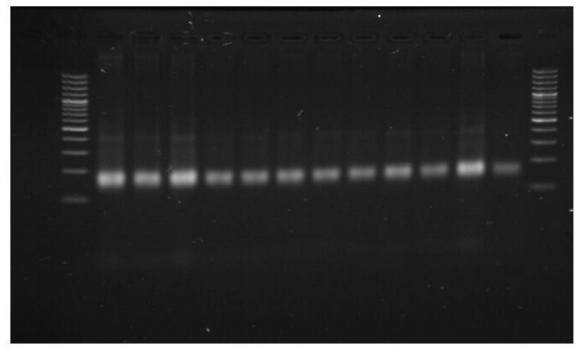

$\mathrm{H}$

Figure 2. Appearance of gel runs with increasing reuse (1-14X). $X=$ times reused. A. $1 X$ recycled gel. B. $3 X$ recycled gel. C. 5X recycled gel. D. 7X recycled gel. E. 9X recycled gel. F. 11X recycled gel. G. 13X recycled gel. H. 14X recycled gel. 
The results clearly demonstrate that with cleaning up, recycled gel can resolve the alleles of microsatellite markers. Nevertheless, the capability of the gel to separate amplified PCR products still diminished with repeated use, and the bands became increasingly blurred at the 11th recycle (see Figure 3) and were considerably worse at the 14th recycle. McMullen (2003) indicated that further refinements may extend the number of Agarose SFR ${ }^{\mathrm{TM}}$ (Amresco) recycles to as many as 40 .
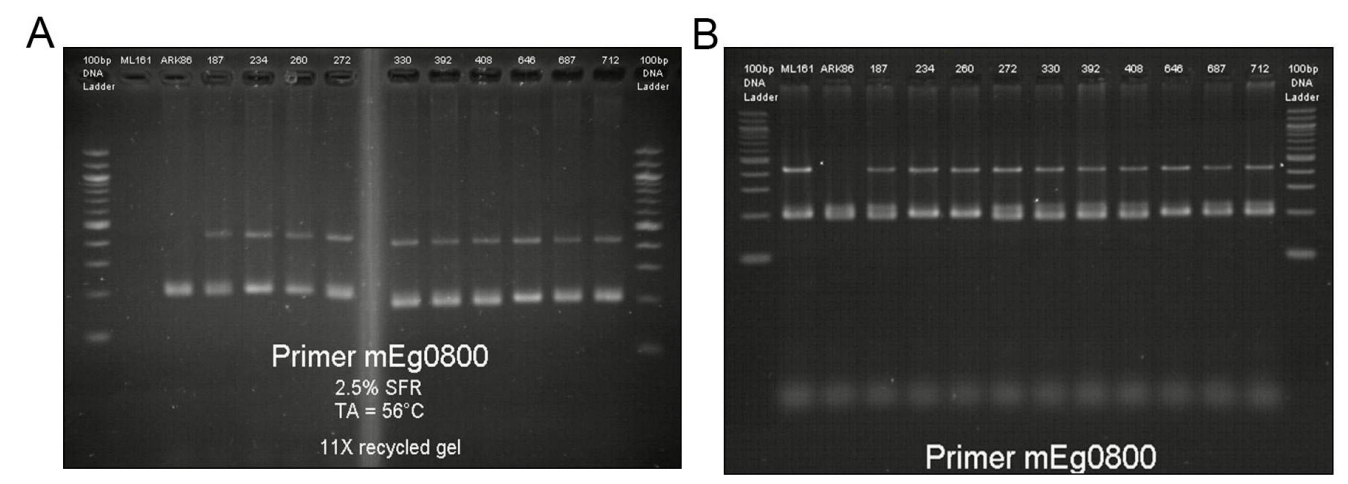

Figure 3. Comparison between fresh and $11 \mathrm{X}$ recycled 2.5\% (w/v) superfine resolution (SFR) gel. A. 11X recycled $2.5 \%(w / v)$ SFR gel. B. Fresh $2.5 \%(w / v)$ SFR gel.

\section{DISCUSSION}

Agarose is non-toxic and easy to store in the laboratory. It melts easily in any running buffer, and the mixture is easy to pour and cast. For these and other reasons, agarose gel has advantages over PA gel systems with silver staining or fluorescent dye labeling in microsatellite primer screening.

Conventionally, buffers such as TAE and TBE are used in gel runs at lower voltages to avoid overheating and melting the gels. The downside is slower gel runs and more time for optimal electrophoretic separation. We found that $1 \mathrm{X}$ LB buffer was amenable to higher voltage $(180 \mathrm{~V})$ electrophoresis, as it remained cool over long periods $(40 \mathrm{~min})$ even at high power. The fragments were adequately separated on the gels, which were cloudless under transillumination. At a higher voltage with $1 \mathrm{X}$ LB buffer, an agarose gel electrophoretic run was completed 4-6 times faster than that using TAE or TBE buffer. In our laboratory, 1 individual can routinely screen 8 primers (12 samples per primer) and run 8 gels per day with a hands-on time of $<3$ h using 3 gel electrophoresis sets.

Using orange-G dye was also advantageous because it runs at the front of the sample at $<50 \mathrm{bp}$ on $2-3 \%$ agarose gel (Table 1). Compared with the commonly used bromophenol blue and xylene cyanine blue, which tend to mask gels at 50-150 and 200-750 bp, respectively, under UV transillumination, orange-G does not obscure DNA samples or PCR products when the gel is examined under UV transillumination. Furthermore, xylene cyanine blue migrates close to the microsatellite fragments with allele sizes that are normally 100-300 bp. The blue dyes also diffuse out of the gels and darken the electrophoresis buffer to a deep blue during each run, thus obscuring the results more than the lighter orange dye does. Clearly, use of orange-G dye prolongs buffer use. 
Table 1. Molecular sizes of dyes used in agarose gel electrophoresis.

\begin{tabular}{lcc}
\hline Dye & $0.5-1.5 \%$ agarose & $2.0-3.0 \%$ agarose \\
\hline Xylene cyanol blue & $>4000 \mathrm{bp}$ & $750-200 \mathrm{bp}$ \\
Bromophenol blue & $500-400 \mathrm{bp}$ & $150-50 \mathrm{bp}$ \\
Orange-G & $<100 \mathrm{bp}$ & $<50 \mathrm{bp}$ \\
\hline
\end{tabular}

The final aspect of our study examined various concentrations of agarose and found that a 2-5\% agarose concentration was optimal for separating DNA fragments of various lengths (Table 2). The microsatellite alleles used were $100-400 \mathrm{bp}$, for which $4 \%$ is appropriate, according to Amresco. However, the preparation of a 3-4\% gel is technically more difficult than that of a $2.5 \%$ SFR gel, which perfectly separated PCR-amplified products of 100 $1000 \mathrm{bp}$ in our experiments. However, the gel needed to be $<4 \mathrm{~mm}$ thick for good resolution; otherwise, the bands were unclear and looked smeared.

Table 2. Recommended product concentrations for optimal separation of different-sized fragments.

\begin{tabular}{lc}
\hline Gel concentration (\% agarose SFR) & Optimal separation size (bp) \\
\hline 2.00 & $500-2000$ \\
3.00 & $100-1000$ \\
4.00 & $50-500$ \\
5.00 & $20-250$ \\
\hline
\end{tabular}

Amresco product information; SFR = superfine resolution agarose gel (preparative or analytical and compatible with in-gel manipulation).

Our experiments showed that $2.5 \%$ Agarose $\mathrm{SFR}^{\mathrm{TM}}$ (Amresco) with 1X LB buffer and orange-G dye loading sufficed for the analysis of amplified PCR products from microsatellite primers with electrophoresis runs completed in $40 \mathrm{~min}$ at $180 \mathrm{~V}$. With the capability of recycling the gel 14 times without special or additional equipment, SFR agarose is much cheaper (US\$0.20/gel run, used 14 times) than other low melting point high-resolution agarose materials. By comparison, PA gel costs approximately US\$5.33 (used only once) and, with silver staining (Bio-Rad Silver Stain Kit, Cat. No. 161-0443, Bio-Rad Laboratories, Inc.), approximately US\$13.67 per gel (used only once). Moreover, its electrophoresis and silver staining take $8 \mathrm{~h}$. Clearly, the agarose SFR system compares favorably with the many PA gels and silver staining techniques widely used for primer screening or genotyping of microsatellite markers. For mapping projects calling for standard protocols using PA gels, our procedures greatly simplify and expedite microsatellite primer screening.

\section{ACKNOWLEDGMENTS}

We thank our respective organizations for support and permission to publish this paper. We especially thank Mohammad Asyraf Md Yusoof and Intan Nur Ainni Mohamed Azni for technical assistance. We also acknowledge a financial grant from Yayasan Felda, which enabled this study.

\section{REFERENCES}

Boffey SA (1984). Agarose Gel Electrophoresis of DNA. In: Nucleic Acids. Vol. 2 (Walker JM, ed.). Humana Press Inc., Totowa, 43-50. 
Eschholz TW, Peter R, Stamp P and Hund A (2008). Genetic diversity of Swiss maize (Zea mays L. ssp. mays) assessed with individuals and bulks on agarose gels. Genet. Resour. Crop Evol. 55: 971-983.

Gaudet M, Fara A-G, Sabatti M, Kuzminsky E, et al. (2007). Single-reaction for SNP genotyping on agarose gel by allelespecific PCR in black poplar (Populus nigra L.). Plant Mol. Biol. Rep. 25: 1-9.

Guilliatt AM (2002). Agarose and Polyacrylamide Gel Electrophoresis. In: PCR Mutation Detection Protocols. Vol. 187 (Theophilus BDM and Rapley R, eds.). Humana Press Inc., Totowa, 1-12.

McMullen MD (2003). Quantitative trait locus analysis as a gene discovery tool. Methods Mol. Biol. 236: 141-154.

Palacios G, Giménez C and García ED (2000). Recycling agarose. Plant Mol. Biol. Rep. 18: 47-49.

Perumal R, Nimmakayala P, Erattaimuthu SR, No EG, et al. (2008). Simple sequence repeat markers useful for sorghum downy mildew (Peronosclerospora sorghi) and related species. BMC Genet. 9: 77.

Raji AA, Anderson JV, Kolade OA, Ugwu CD, et al. (2009). Gene-based microsatellites for cassava (Manihot esculenta Crantz): prevalence, polymorphisms, and cross-taxa utility. BMC Plant Biol. 9: 118.

Routman E and Cheverud J (1994). A rapid method of scoring simple sequence repeat polymorphisms with agarose gel electrophoresis. Mamm. Genome 5: 187-188.

Wang D, Shi J, Carlson SR, Cregan PB, et al. (2003). A low-cost, high-throughput polyacrylamide gel electrophoresis system for genotyping with microsatellite DNA markers. Crop Sci. 43: 1828-1832.

White HW and Kusukawa N (1997). Agarose-based system for separation of short tandem repeat loci. Biotechniques 22: 976-980. 